\title{
Theory of Polymers in Poor Solvent: Phase Equilibrium and Nucleation Behavior
}

\author{
Rui Wang and Zhen-Gang Wang* \\ Division of Chemistry and Chemical Engineering, California Institute of Technology, Pasadena, California 91125, United States
}

ABSTRACT: We study the phase equilibrium and nucleation behavior of polymers in poor solvent by accounting for the large, localized fluctuations in the form of single-chain globules and multichain clusters. The density profile and free energy of the single-chain globule and multichain clusters are obtained by self-consistent-field theory. This information is then used in the dilute solution thermodynamics to investigate the equilibrium cluster size distribution, solubility limit, and nucleation in the supersaturated state. Our results show that the solubility of the polymer in the dilute side of the solution is enhanced by several orders of magnitude relative to the prediction of the Flory-Huggins $(\mathrm{F}-\mathrm{H})$ theory, which scales with the chain length to the $2 / 3$ power rather than a linear power as predicted from the $\mathrm{F}-\mathrm{H}$ theory. Our results also suggest a higher critical value of $\chi$, consistent with computer simulation and experiment results. In the supersaturated state, we work out an effective spinodal where the nucleation barrier to phase separation via growth of the clusters becomes comparable to the thermal energy $k_{\mathrm{B}} T$. For a given supersaturation, we find that the nucleation barrier is quadratic in the chain length, suggesting a much slower precipitation rate for longer polymer chains.

\section{INTRODUCTION}

Polymer solutions are widely used in biomedical, pharmaceutical, food, and cosmetics industries. ${ }^{1-4} \mathrm{~A}$ basic question is whether a prepared polymer solution under a given operation condition is stable. The most widely used framework to describe the thermodynamics of polymer solutions is the Flory-Huggins $(\mathrm{F}-\mathrm{H})$ theory. ${ }^{5,6}$ It is a mean-field theory, which assumes random mixing of ideal chains and solvent molecules, and characterizes the polymer-solvent interaction by a phenomenological parameter $\chi^{7,8}$ The $\mathrm{F}-\mathrm{H}$ theory predicts a coexistence of polymer concentrated phase and polymer dilute phase above the critical $\chi$ (or equivalently below the critical temperature). For polymer chains of length $N$, the critical $\chi$ and the critical polymer concentration are $1 / 2+N^{-1 / 2}$ and $N^{-1 / 2}$, respectively. From the $\mathrm{F}-\mathrm{H}$ theory, des Cloizeaux and Jannink ${ }^{2}$ obtained the equilibrium polymer concentration in the dilute phase well below the critical point as $\phi_{\mathrm{L}}=(3 /$ e) $(1 / 2-\chi) \exp [-3 / 2(1 / 2-\chi) N]$. The phase diagram calculated by the $\mathrm{F}-\mathrm{H}$ theory shows poor agreement with experiments and simulation for the dilute phase and near the critical point: the $\mathrm{F}-\mathrm{H}$ theory predicts a much lower equilibrium polymer concentration in the dilute phase and a lower critical value of $\chi .^{9-12}$ Many modifications have been made to the $\mathrm{F}-\mathrm{H}$ theory, such as treating $\chi$ as a function of concentration, temperature, and chain length, ${ }^{13-15}$ accounting for the local enrichment of the chain segments due to chain connectivity, ${ }^{16}$ and performing a perturbation expansion in the interaction strength and the inverse of the lattice coordination number. ${ }^{17-19}$

An obvious fact that is not reflected in the $\mathrm{F}-\mathrm{H}$ theory is the large, local concentration fluctuation in the dilute phase, which originates from two physical mechanisms. First, a polymer chain is a connected object, so an instantaneous picture of the solution will have localized high concentrations where the chains are located and pure solvents elsewhere; this is a very different scenario than envisioned in the random mixing pictured of the $\mathrm{F}-\mathrm{H}$ theory. Second, the polymer chains will form compact globules in the poor solvent as demonstrated by numerous experiments and simulations. ${ }^{20-23}$ Globules can further aggregate to larger clusters. In a series of papers, Grosberg and Kuznetsov ${ }^{24-27}$ investigated the coil-to-globule transition, the globule-globule interaction, and the resulting phase equilibrium by using the Lifshitz theory, ${ }^{28}$ which assumes ground-state dominance and viral expansion of the local interaction. In this work, we use a different approach to study the solution behavior in the poor solvent. First, instead of the ground-state dominance method, we employ the full selfconsistent-field theory (SCFT) which affords a more accurate description of the density profile of the globule and its free energy for finite chain lengths. ${ }^{29,30}$ Second, we describe our model and results in the language of $\mathrm{F}-\mathrm{H}$ theory in terms of the $\chi$ parameter and treating the solution as incompressible, thus allowing a direct comparison with the predictions from the $\mathrm{F}-\mathrm{H}$ theory for the phase diagram. Third, rather than treating the interaction between globules in a pairwise manner, we include clusters of different sizes to account for the aggregation between the globules. And finally, we consider the nucleation of polymer precipitation beyond the coexistence limit, which can be naturally studied with the clusters approach.

Received: May 22, 2012

Revised: July 14, 2012

Published: July 24, 2012 


\section{MODEL}

We consider a closed system of homopolymers of $N$ Kuhn segments in a poor solvent. For simplicity, the volume of the solvent molecule and the chain segment are assumed to be the same $v_{0}$. The polymer chains can form clusters of different aggregation number $m$ (called $m$-cluster henceforth). $m=1$ is the single-chain globule. We investigate the fluctuation structure of the solution by focusing on the formation of a single isolated cluster in a chosen subvolume. Anticipating the low concentration of polymers in the polymer-poor phase well below the critical point, we ignore the interaction between different clusters. The free energy density of the system, including the translational entropy of the clusters, can be written as

$$
F / V=\sum_{m=1}^{\infty}\left\{C_{m} F_{m}+k_{\mathrm{B}} T C_{m}\left[\ln \left(C_{m} a_{m}\right)-1\right]\right\}
$$

where $C_{m}$ is the concentration of the $m$-cluster and $F_{m}$ is the standard free energy of an isolated $m$-cluster situated with a fixed center-of-mass position. $a_{m}$ in eq 1 is a reference volume which arises from the evaluation of the full partition function for the cluster and cannot be determined from a mean-field theory such as SCFT. ${ }^{31,32}$ Aside from setting a length scale for the concentration, the role of $a_{m}$ is insignificant in the thermodynamics of the system; therefore, for simplicity, we take it to be the volume of the $m$-cluster, and thus $C_{m} a_{m}$ becomes just the volume fraction of the $m$-cluster. The free energy of a single $m$-cluster along with its density profile is calculated numerically using SCFT in semicanonical ensemble by focusing on a subvolume large enough to fully contain one cluster but small enough so the probability of having more than one cluster is negligible. No preassumption of the cluster structure other than spherical symmetry is included in the calculation. We note that in other contexts, for example, micelles formed by block copolymers, questions have been raised as to the validity of using SCFT, ${ }^{39}$ which does not account for soft-mode fluctuations, such as shape fluctuation of the micelles. However, well below the theta temperature, as is the case of interest in our work, the interfacial tension between the globule and the solvent is sufficiently high to maintain the shape and density profile of the globule, and thus SCFT should be well suited to study the properties of a single-chain globule and multichain aggregates. We refer the interested readers to the relevant literature for the formulation and numerical details of SCFT. ${ }^{29,30}$

\section{SINGLE-CHAIN GLOBULES AND MULTICHAIN CLUSTERS}

The $\mathrm{F}-\mathrm{H} \chi$ parameter in this work is chosen to be far from the $\theta$ region $\left(\chi>\chi_{\mathrm{c}}, \chi_{\mathrm{c}}\right.$ is the critical value of Flory-Huggins parameter at the $\theta$ point) to ensure the polymers are in globule or cluster state. Because of the unfavorable interaction with the solvent, chain segments tend to compact themselves to reduce their contact with the solvent molecules. The increase in local segment density is countered by excluded volume (modeled here by the incompressibility) which prevents the globule or cluster structure from collapsing into unbounded high density. The density profile of a globule is shown in Figure 1 for several values of $\chi$. The globule structure can be divided into a core region with uniform density and an interfacial region. As $\chi$ decreases, the globule core becomes smaller and has lower polymer density while the interface becomes wider and more

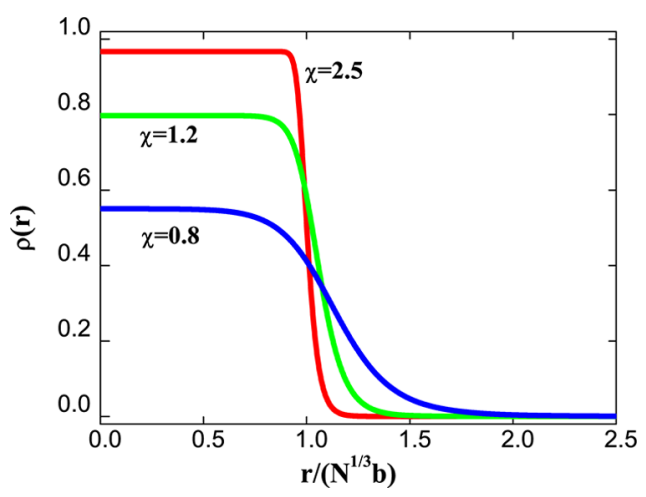

Figure 1. Effect of $\mathrm{F}-\mathrm{H} \chi$ parameter on density profile of globule. $\rho(r)$ is the local volume fraction of polymer. $b$ is the Kuhn length of chain segments. $N=100$.

diffuse. Although the solution itself is dilute, the globule core is quite dense, and only a small amount of solvent molecules can penetrate into the globule core. Given the spatial structure of the globule, it is obvious that polymer-solvent interaction is not uniform as envisioned in the random mixing approximation in the $\mathrm{F}-\mathrm{H}$ theory but is primarily concentrated in the interfacial region.

Clusters of higher aggregation number have the same structure as a single-chain globule except for the larger core. Grosberg and Kuznetsov ${ }^{24}$ point out that the globule core contains a number of uncorrelated parts of the chain, which resembles a concentrated solution of independent polymer chains. Approximating the core as a concentrated solution, SCFT is simplified to $\mathrm{F}-\mathrm{H}$ theory, which is an accurate description for concentrated polymer solutions. The polymer density of the cluster core can be evaluated by balancing the osmotic pressure inside the cluster with that of the pure solvent outside (i.e., zero), as

$$
\left(1-\frac{1}{N}\right) \rho+\ln (1-\rho)+\chi \rho^{2}=0
$$

The nontrivial root of eq 2 , denoted by $\rho_{\infty}$, is the polymer density for an infinitely large cluster. As shown in Figure 2a, the actual polymer density at the center of a $m$-cluster $\rho_{m}$ (calculated by the full SCFT) is higher than $\rho_{\infty}$; this is consistent with the effect of the Laplace pressure in the

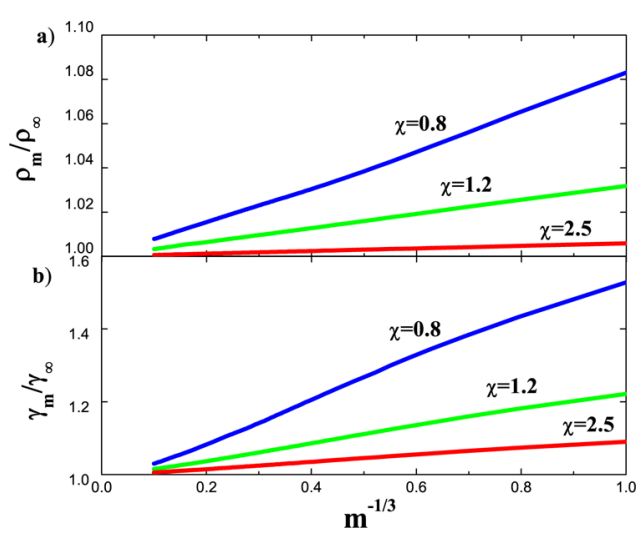

Figure 2. Dependence of (a) core density $\rho_{m}$ and (b) interfacial free energy $\gamma_{m}$ on cluster size. $N=100 . \rho_{m}$ and $\gamma_{m}$ are calculated from SCFT, $\rho_{\infty}$ is the nontrivial root of eq 2 , and $\gamma_{\infty}$ is obtained by a separate SCFT calculation for a planar interface. 
spherical droplet due to the interfacial tension. $\rho_{m}$ approaches $\rho_{\infty}$ as $m$ increases, roughly linearly with the cluster curvature $\left(\sim m^{-1 / 3}\right)$. The approach is faster for larger values of $\chi$.

As shown in Figure $2 \mathrm{~b}$, the interfacial free energy of the $m$ cluster, $\gamma_{m}$, is higher than the interfacial tension of the planner interface (denoted by $\gamma_{\infty}$ ) due to the curvature correction of the interfacial tension. $\gamma_{m}$ approaches $\gamma_{\infty}$ with the increase of $m$ and $\chi$. Based on the cluster structure, the free energy of the $m$ cluster, $F_{m}$, can be approximated by adding the core term and the interface term:

$$
F_{m} \approx f_{\mathrm{FH}}\left(\rho_{m}\right) V_{m}+\gamma_{m} A_{m}
$$

where $f_{F H}\left(\rho_{m}\right)$ is the $\mathrm{F}-\mathrm{H}$ free energy density evaluated at polymer density $\rho_{m} . V_{m}=m N v_{0} / \rho_{m}$ and $A_{m}=\left(6 \pi^{1 / 2} m N v_{0} /\right.$ $\left.\rho_{m}\right)^{2 / 3}$ are the volume and the surface area of the $m$-cluster, respectively. For clusters with very large aggregation number, eq 3 provides a simple approximation of the cluster free energy by using $\rho_{\infty}$ and $\gamma_{\infty}$ in place of $\rho_{m}$ and $\gamma_{m}$; for small clusters (especially the globule), the free energy is obtained with the full SCFT.

The equilibrium concentration of the $m$-clusters in the system can be obtained by minimization of the free energy density in eq 1 subject to a fixed total polymer concentration $\sum_{m=1}^{\infty} m C_{m}$, which leads to

$$
F_{m}+k_{\mathrm{B}} T \ln \phi_{m}=m \mu
$$

where $\phi_{m}$ is the volume fraction of the $m$-cluster. $\mu$ is the Lagrangian multiplier ensuring mass conservation, which can be interpreted as the chemical potential of a polymer chain. Equation 4 thus is equivalent to equality of the chemical potential of the polymer chain in all clusters. Expressing $\mu$ in terms of the globule properties (i.e., $\mu=F_{1}+k_{B} T \ln \phi_{1}$ ) results in the following cluster distribution:

$$
\phi_{m}=\left(\phi_{1}\right)^{m} \exp \left(-\frac{\Delta F_{m}}{k_{\mathrm{B}} T}\right)
$$

where $\Delta F_{m} \equiv F_{m}-m F_{1}$ is the free energy of formation of the $m$-cluster from $m$ single-chain globules. Based on eq $3, \Delta F_{m}$ is proportional to $\left(m^{2 / 3}-m\right) N^{2 / 3} \gamma_{m}$ if we neglect the cluster size dependence of $\rho_{m}$ and $\gamma_{m}$. This simple formula explains the behavior of $\Delta F_{m}$ shown in Figure 3: $\Delta F_{m}$ decays monotonically with the increase of $m$ and becomes more negative as either $\chi$ increases (thus increasing $\gamma_{m}$ ) or $N$ increases.

According to eq 5 , the cluster distribution is determined by the competition between the formation free energy $\Delta F_{m}$ and the translational entropy (manifested in $\left.\left(\phi_{1}\right)^{m}\right)$. Larger cluster is energetically preferable, while translational entropy favors single-chain globules. In a stable dilute solution, the entropic effect dominates, and thus single-chain globules occupy the largest volume fraction in the cluster distribution as shown in Figure 4. $\phi_{m}$ decreases with increasing $m$. The volume fraction of larger clusters increases as $\phi_{1}$ increases (i.e., approaching the phase coexistence curve). Further increasing $\phi_{1}$ past the coexistence curve, the solution becomes metastable. In this regime, globules will aggregate and eventually precipitate from the poor solvent, which we will discuss in section V.

\section{SOLUBILITY LIMIT}

The free energy and distribution of clusters obtained above allow us to construct an improved phase diagram for polymer chains in poor solvents. The $\mathrm{F}-\mathrm{H}$ theory significantly overestimates the polymer-solvent interaction in dilute

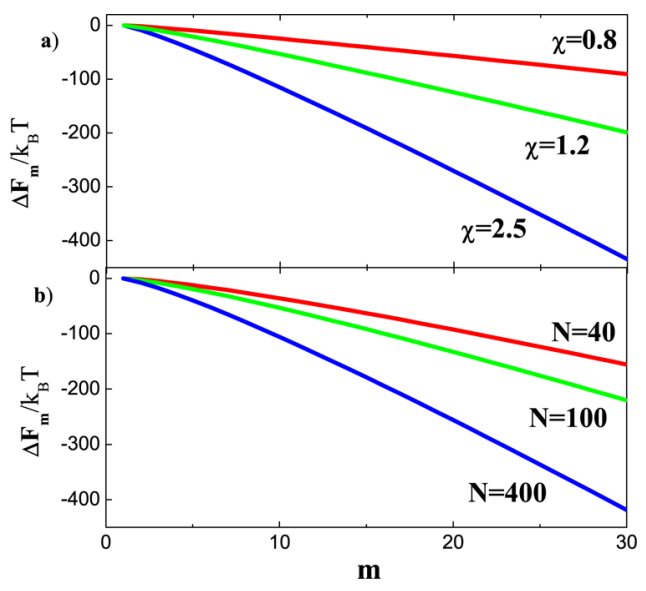

Figure 3. Standard formation energy of clusters versus the aggregation number $m$ : (a) for three levels of $\chi$ at $N=100$; (b) for three levels of $N$ at $\chi=1.0$.

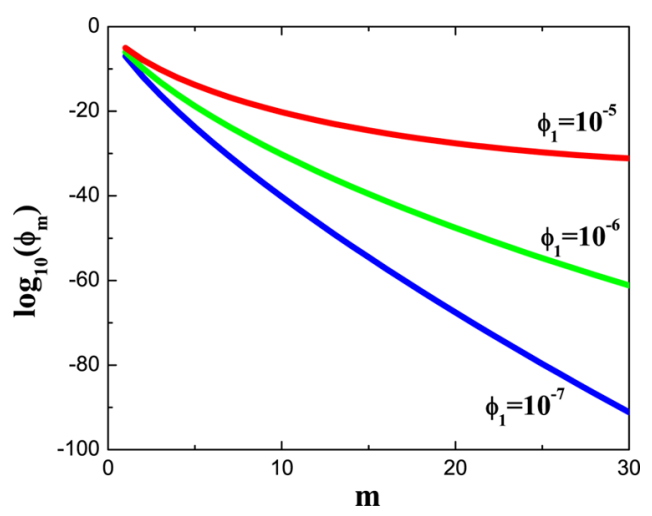

Figure 4. Volume fraction of clusters versus the aggregation number $m$ for three values of $\phi_{1} \cdot \chi=1.0, N=100 . \phi_{1}$ at phase equilibrium is 9.7 $\times 10^{-5}$.

phase, and thus the calculated phase coexistence curve is very much off. In this work, we use the free energy in eq 1 to account for the large, local fluctuation structure of the dilute polymer solution, whereas the $\mathrm{F}-\mathrm{H}$ theory is used in the concentrated phase. Grosberg and Kuznetsov included only globules in their calculation of phase equilibrium. They accounted for the pair interaction between globules and found that aggregation can lower the system free energy. ${ }^{26}$ Here, we allow the existence of larger clusters and determine the coexistence by the respective equality of the chemical potential of the polymer and the solvent in the two coexisting phases:

$$
\begin{aligned}
& \frac{F_{1}}{k_{\mathrm{B}} T}+\ln \phi_{1}= \ln \phi_{\mathrm{H}}+\left(1-\frac{1}{N}\right)\left(1-\phi_{\mathrm{H}}\right) \\
&+\chi N\left(1-\phi_{\mathrm{H}}\right)^{2} \\
&-\sum_{m=1}^{\infty} \frac{\phi_{m}^{*}}{m N}=\left(1-\frac{1}{N}\right) \phi_{\mathrm{H}}+\ln \left(1-\phi_{\mathrm{H}}\right)+\chi \phi_{\mathrm{H}}{ }^{2}
\end{aligned}
$$

where $\phi_{\mathrm{H}}$ is the equilibrium volume fraction of polymers in concentrated phase and $\phi_{m}^{*}$ is the equilibrium volume fraction of the $m$-cluster in the dilute phase given by eq 5 . We recognize the sum on the left-hand side of eq $6 \mathrm{~b}$ as the (dimensionless) osmotic pressure in the dilute phase (in accordance with the 
van't Hoff law), as expected for an ideal dilute solution. Because $\phi_{m}$ decreases rapidly with $m$ (as shown in Figure 4), the summation is dominated by the single-chain globules with some significantly reduced contributions from dimers and trimers; we thus include clusters sizes only up to 3 . The resulting coexistence curve for the dilute phase is shown in Figure 5. For the concentrated phase, the coexistence curve is very close to that calculated by the $\mathrm{F}-\mathrm{H}$ theory, which is not shown here.

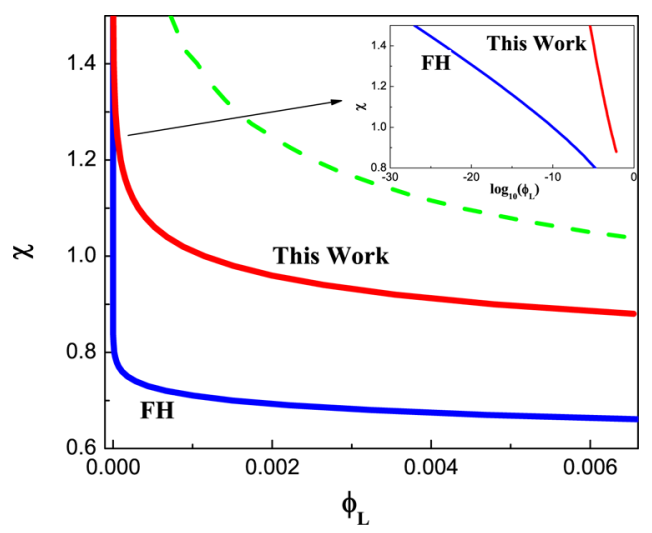

Figure 5. Phase boundary on the dilute side of the coexistence for $N=$ 100. $\phi_{\mathrm{L}}$ is the equilibrium polymer concentration in the dilute phase $\left(\phi_{\mathrm{L}}=\sum_{m=1}^{\infty} \phi_{m}^{*}\right.$. The lines denoted by "This Work" and "FH" are the results calculated by our model and by the $\mathrm{F}-\mathrm{H}$ theory, respectively. The inset uses logarithmic scale of $\phi_{\mathrm{L}}$ to illustrate the huge difference between the results of the two models at large $\chi$. The dashed line is the pseudospinodal line discussed in section V.

Because the $\mathrm{F}-\mathrm{H}$ theory overestimates the penalty of polymer-solvent interaction, it predicts a much lower solubility of polymers in a poor solvent compared with experiments and simulation. By including large, localized fluctuation in the form of globules and clusters, the coexistence volume fraction of polymers in the dilute phase calculated by our model is much higher than predicted by the $\mathrm{F}-\mathrm{H}$ theory, as shown in Figure 5. The improvement can be as large as tens of order of magnitude with the increase of $\chi$. Although our calculation cannot be extended to the critical region, it can be seen from the trend of the coexistence curve that the critical $\chi$ extrapolated from our model would be higher than that predicted by the $\mathrm{F}-\mathrm{H}$ theory, in agreement with the experiment and simulation results. ${ }^{9,10,33}$

In addition to the large quantitative discrepancy in the phase boundary, our model also yields different scaling behavior for the equilibrium concentration of polymers in the dilute phase $\left(\phi_{\mathrm{L}}=\sum_{m=1}^{\infty} \phi_{m}^{*}\right)$ with the polymer chain length $N$. Using the F$\mathrm{H}$ theory, des Cloizeaux and Jannink ${ }^{2}$ obtained $\phi_{\mathrm{L}}=(3 / e)(1 / 2$ $-\chi) \exp [-3 / 2(1 / 2-\chi) N]$; i.e., $\ln \phi_{\mathrm{L}}$ scales linearly with $N$. In our model, $\ln \phi_{\mathrm{L}}$ decreases linearly with $N^{2 / 3}$, as shown in Figure 6 . The different $\mathrm{N}$-dependence arises from the fact that the $\mathrm{F}-\mathrm{H}$ theory assumes random, uniform mixing between the solvent and the chain segments whereas the solvent-polymer contact in our model mainly takes place at the cluster surface. Moreover, the slope of $\ln \phi_{\mathrm{L}}$ versus $N^{2 / 3}$ is a function of $\chi$ and becomes more negative with increasing $\chi$, as shown in Figure 6 . By measuring the slope of $\ln \phi_{\mathrm{L}}$ versus $N^{2 / 3}$, our model suggests a method for determining $\chi$ from experiment.

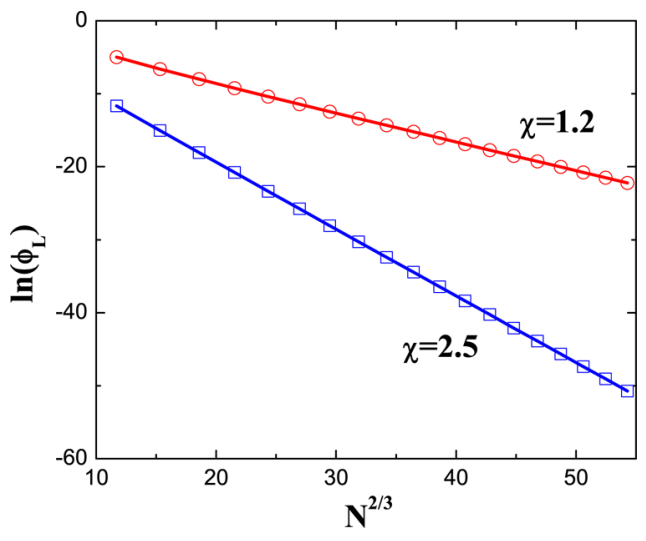

Figure 6. Linear relation between $\ln \phi_{\mathrm{L}}$ and $N^{2 / 3}$.

\section{NUCLEATION IN THE SUPERSATURATED STATE}

Above the phase boundary in Figure 5, the solution becomes supersaturated with polymers. Polymer chains will precipitate from the solvent to form the polymer-rich phase through the nucleation and growth mechanism. ${ }^{35}$ The precipitation rate is related to the free energy barrier to forming the critical nucleus (the cluster with critical aggregation number $m^{*}$ ). The formation free energy of an $m$-cluster from $m$ free globules, $\Delta G_{m}$, is given by

$$
\Delta G_{m}=F_{m}-m \mu=\Delta F_{m}-k_{\mathrm{B}} T \ln \phi_{1}
$$

Figure 7 shows a typical energy curve as a function of the cluster size. If we take the cluster size $m$ as the "reaction

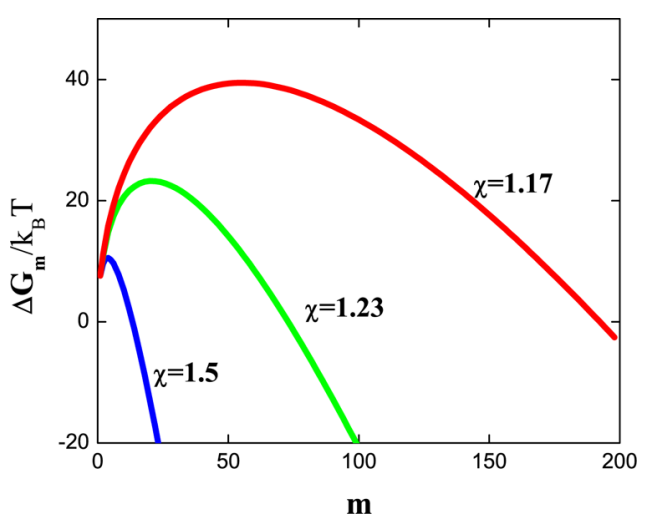

Figure 7. Free energy to form a cluster from free globules as a function of the aggregation number. $\phi_{1}$ is fixed at $5 \times 10^{-4} . N=100$.

coordinate", the figure can be interpreted as the free energy path for cluster growth. The maximum of the free energy gives the size of the critical nucleus $\left(m^{*}\right)$ and the nucleation barrier $\left(\Delta G_{m *}\right)$.

The size of the critical nucleus and nucleation barrier as a function of $\chi$ at fixed concentration of the free globules are shown in Figure 8. Both $m^{*}$ and $\Delta G_{m}{ }^{*}$ diverge at the phase coexistence curve, as demanded by thermodynamic consistency. $m^{*}$ and $\Delta G_{m}{ }^{*}$ decrease rapidly as $\chi$ increases away from the coexistence curve, reflecting the fact that the metastable solution becomes less stable. For our system, $\Delta G_{m}{ }^{*}$ becomes less than $10 k_{\mathrm{B}} T$ (with $m^{*} \approx 3$ ) when $\chi>1.55$. When the nucleation barrier becomes comparable to $k_{\mathrm{B}} T$, the lifetime of the metastable state becomes sufficiently short that an equilibrium description of the system becomes questionable. 


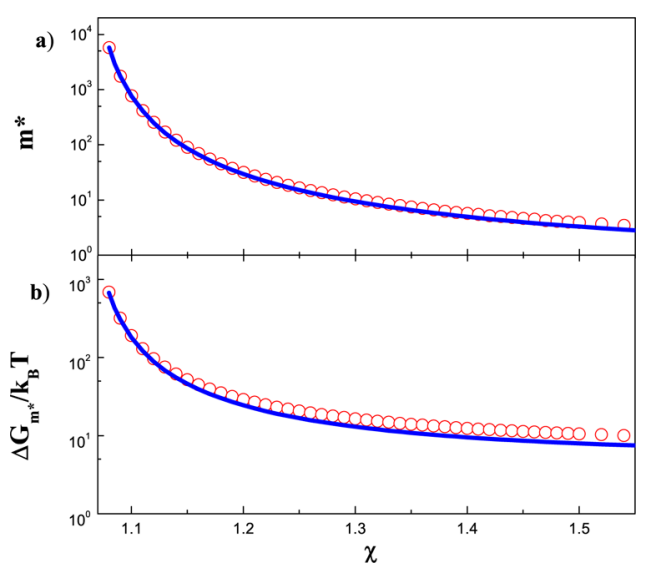

Figure 8. (a) Size of critical nucleus and (b) the nucleation energy barrier as a function of $\chi . \phi_{1}$ is fixed at $5 \times 10^{-4} . N=100 . \chi$ at phase coexistence curve is 1.07 . Circles and solid lines are the results obtained by the exact method and the approximate method, respectively.

In addition, in this regime the concentration of clusters is no longer small to justify ignoring their interactions. Nevertheless, one may define an operational limit of metastability (what has been termed the pseudospinodal ${ }^{34}$ ) when the nucleation barrier is $10 k_{\mathrm{B}} T$. This pseudospinodal line is shown as the dashed curve in Figure 5.

We can obtain approximate analytical expressions for the critical nucleus size and the nucleation barrier by using the approximate expression for $\Delta F_{m}$, eq 3 , in eq 7 and subsequently replacing $\rho_{\infty}$ and $\gamma_{\infty}$ with $\gamma_{m}$ and $\rho_{m} . \Delta G_{m}$ now becomes

$$
\begin{aligned}
\Delta G_{m} \approx & f_{\mathrm{FH}}\left(\rho_{m}\right) V_{m}+\gamma_{m} A_{m}-m F_{1}-m k_{\mathrm{B}} T \ln \phi_{1} \\
\approx & {\left[f_{\mathrm{FH}}\left(\rho_{\infty}\right) N v_{0} / \rho_{\infty}-F_{1}-k_{\mathrm{B}} T \ln \phi_{1}\right] m } \\
& +\gamma_{\infty}\left(6 \pi^{1 / 2} N v_{0} / \rho_{\infty}\right)^{2 / 3} m^{2 / 3}
\end{aligned}
$$

Clearly, $\Delta G_{m}$ contains a volume term $(\sim m)$ and a surface term $\left(\sim m^{2 / 3}\right)$; this is just the classical nucleation theory $(\mathrm{CNT}){ }^{35,36}$ The critical nucleus is obtained by $\partial \Delta G_{m} / \partial m=0$, leading to

$$
\begin{aligned}
& m^{*}=\frac{32 \pi\left(N v_{0}\right)^{2} \gamma_{\infty}{ }^{3}}{3 \rho_{\infty}{ }^{2}\left[F_{1}+k_{\mathrm{B}} T \ln \phi_{1}-f_{\mathrm{FH}}\left(\rho_{\infty}\right) N v_{0} / \rho_{\infty}\right]^{3}} \\
& \Delta G_{m *}=\frac{8 \pi\left(N v_{0}\right)^{2} \gamma_{\infty}{ }^{3}}{3 \rho_{\infty}{ }^{2}\left[F_{1}+k_{\mathrm{B}} T \ln \phi_{1}-f_{\mathrm{FH}}\left(\rho_{\infty}\right) N v_{0} / \rho_{\infty}\right]^{2}}
\end{aligned}
$$

As shown in Figure 8, these analytical expressions, which only require the free energy of the globule $F_{1}$, the bulk concentration $\rho_{\infty}$, and interfacial tension $\gamma_{\infty}$, are good approximations to the results obtained using the full SCFT. Therefore, whereas the classical nucleation theory is known to be a poor description for small-molecule systems, for the precipitation of polymers in poor solvents, CNT provides a good description of the nucleation behavior.

It is instructive to express $m^{*}$ and $\Delta G_{m}{ }^{*}$ in terms of supersaturation $S . S$ in our case is defined as $\phi_{1} / \phi_{1}^{*}$, with $\phi_{1}^{*}$ the volume fraction at phase coexistence (where $m^{*}$ in eq 9 diverges). $S$ measures the relative distance from the phase coexistence and can be considered the driving force to form the new phase from the metastable solution. Based on the definition of $S$, eqs 9 and 10 can be rearranged to give the following scaling behaviors:

$$
\begin{aligned}
& m^{*} \sim \gamma_{\infty}{ }^{3} \rho_{\infty}{ }^{-2} N^{2}\left(k_{\mathrm{B}} T \ln S\right)^{-3} \\
& \Delta G_{m *} \sim \gamma_{\infty}{ }^{3} \rho_{\infty}{ }^{-2} N^{2}\left(k_{\mathrm{B}} T \ln S\right)^{-2}
\end{aligned}
$$

The dependence on the supersaturation $S$ is consistent with the CNT. ${ }^{35,36}$ Importantly, however, $\Delta G_{m}{ }^{*}$ shows a strong dependence with the chain length as $N^{2}$ (for sufficiently long chains, $\rho_{\infty}$ and $\gamma_{\infty}$ become independent of $N$ ). At the same supersaturation, the nucleation barrier increases with chain length. Equation 12 can also be viewed in another perspective: the supersaturation required to reach the pseudospinodal (by setting the right-hand side of eq 12 to $10 k_{\mathrm{B}} T$ ) increases exponentially with the molecular weight. Therefore, it is more difficult to precipitate polymers than small molecules; polymer precipitation requires a much higher supersaturation.

\section{CONCLUSIONS}

In this work, we have studied the phase equilibrium and nucleation behavior of a dilute solution of polymer chains in poor solvents by taking into account the large, localized fluctuations in the form of globules and clusters. The free energy and the density profile of the single-chain globule and multichain clusters are obtained by the SCFT. This information is then used in the framework of the dilute solution thermodynamics to predict the equilibrium cluster size distribution, solubility limit, and nucleation in the supersaturated state. Our theory yields a much higher solubility limit (by several orders of magnitude) for the polymer than that given by the $\mathrm{F}-\mathrm{H}$ theory and also suggests a higher critical value of $\chi$ for the phase diagram. Moreover, our theory predicts different scaling between the equilibrium concentration of polymers at the solubility limit $\phi_{\mathrm{L}}$ and the chain length $N: \ln \phi_{\mathrm{L}}$ decreases linearly with $N^{2 / 3}$ instead of $N$. In addition, by examining the nucleation barrier in the supersaturated state, we obtain the physical limit of metastability-the pseudospinodal-at which the barrier becomes comparable to the thermal energy. For a given supersaturation, we find that the nucleation barrier is proportional to $N^{2}$, which suggests a slower precipitation rate of longer polymer chains.

We close with two perspective remarks. First, the effects we study in this work are clearly due to large fluctuations that cannot be captured by the usual mean-field theory. However, when the fluctuations are local and uncorrelated-such as in the form of isolated globules and clusters studied here-it is possible to address the non-mean-field effects with a mean-field theory (the SCFT) if we judicially identify and isolate these local fluctuations. ${ }^{31,37,38}$ We note that if we applied the SCFT on the polymer solution as a whole, we would merely recover the $\mathrm{F}-\mathrm{H}$ theory. Wang et al. ${ }^{31}$ used a similar strategy to study the effects of disordered micelles on the order-disorder transition in asymmetric diblock copolymer melts. We thus advocate such a strategy as a simple and effective means for studying large, localized fluctuation effects in polymeric systems.

Second, from the point of view of nucleation theory, our results show that the classical nucleation theory provides a good description of the nucleation in the precipitation of the polymers in supersaturated state. For nucleation in smallmolecule systems, such as condensation of supersaturated vapor, or vaporization of superheated liquids, some of the key assumptions concerning the nuclei, such as the spherical shape and uniform, bulklike core, do not hold for small nuclei. ${ }^{35}$ For polymers in poor solvents, however, even a single chain takes 
the form of a spherical globule with a fairly uniform core when the chain is long enough. Therefore, the system studied in this work is as close as one can get to an ideal case for the application of the classical nucleation theory.

\section{AUTHOR INFORMATION}

\section{Corresponding Author}

*E-mail: zgw@caltech.edu.

\section{Notes}

The authors declare no competing financial interest.

\section{ACKNOWLEDGMENTS}

This work is in part supported by the National Science Foundation through MRSEC-CALTECH, and in part supported by NSF Grant No. CHE-1040558.

\section{REFERENCES}

(1) Flory, P. J. Principles of Polymer Chemistry; Cornell University Press: Ithaca, NY, 1953.

(2) des Cloizeaux, J.; Jannink, G. Polymers in Solution. Their Modelling and Structure; Clarendon Press: Oxford, 1990.

(3) Fujita, H. Polymer Solutions; Elsevier: New York,1990.

(4) Hamley, I. W. Block Copolymers in Solution: Fundamentals and Applications; John Wiley \& Sons, Ltd.: Chichester, 2005.

(5) Flory, P. J. J. Chem. Phys. 1942, 10, 51-61.

(6) Huggins, M. L. J. Am. Chem. Soc. 1942, 64, 1712-1719.

(7) de Gennes, P. G. Scaling Concept in Polymer Physics; Cornell University Press: Ithaca, NY, 1993.

(8) Rubinstein, M.; Colby, R. H. Polymer Physics; Oxford University Press: Oxford, 2003.

(9) Takano, N.; Einaga, Y.; Fujita, H. Polym. J. 1985, 17, 1123-1130.

(10) Madden, W. G.; Pesci, A. I.; Freed, K. F. Macromolecules 1990, 23, 1181-1191.

(11) Yan, Q. Y.; Liu, H. L.; Hu, Y. Macromolecules 1996, 29, 40664071.

(12) Panagiotopoulos, A. Z.; Wong, V.; Floriano, M. A. Macromolecules 1998, 31, 912-918.

(13) Koningsveld, R.; Kleintjens, L. A. Macromolecules 1971, 4, 637641.

(14) Koningsveld, R.; Stockmayer, W. H.; Kennedy, J. W.; Kleintjens, L. A. Macromolecules 1974, 7, 73-79.

(15) Einaga, Y.; Ohashi, S.; Tong, Z.; Fujita, H. Macromolecules 1984, $17,527-534$.

(16) Guggenheim, E. A. Proc. R. Soc. London, Ser. A 1944, 183, 203212, 213-227.

(17) Freed, K. F. J. Phys. A 1985, 18, 871-887.

(18) Bawendi, M. G.; Freed, K. F. J. Chem. Phys. 1986, 84, 70367047; 1987, 86, 3720-3730; 1988, 88, 2741-2756.

(19) Freed, K. F.; Bawendi, M. G. J. Phys. Chem. 1989, 93, 21942203

(20) Nishio, I.; Sun, S. T.; Swislow, G.; Tanaka, T. Nature 1979, 281, 208-209.

(21) Wu, C.; Wang, X. Phys. Rev. Lett. 1998, 80, 4092-4094.

(22) Hu, W. J. Chem. Phys. 1998, 109, 3686-3690.

(23) Guo, J.; Liang, H.; Wang, Z.-G. J. Chem. Phys. 2011, 134, art. no. 244904.

(24) Grosberg, A. Yu.; Kuznetsov, D. V. Macromolecules 1992, 25, 1970-1979.

(25) Grosberg, A. Yu.; Kuznetsov, D. V. Macromolecules 1992, 25, 1980-1990.

(26) Grosberg, A. Yu.; Kuznetsov, D. V. Macromolecules 1992, 25, 1991-1995.

(27) Grosberg, A. Yu.; Kuznetsov, D. V. Macromolecules 1992, 25, 1996-2003.

(28) Lifshitz, I. M.; Grosberg, A. Yu.; Khokhlov, A. R. Rev. Mod. Phys. 1978, 50, 683-713.
(29) Fredrickson, G. H. The Equilibrium Theory of Inhomogeneous Polymers; Oxford University Press: Oxford, 2006.

(30) Fredrickson, G. H.; Ganesan, V.; Drolet, F. Macromolecules 2002, 35, 16-39.

(31) Wang, J.; Wang, Z.-G.; Yang, Y. Macromolecules 2005, 38, 1979-1988.

(32) Thiagarajan, R.; Morse, D. C. J. Phys.: Condens. Matter 2011, 23, art. no. 284109.

(33) Szleifer, I. J. Chem. Phys. 1990, 92, 6940-6952.

(34) Wang, Z.-G. J. Chem. Phys. 2002, 117, 481-500.

(35) Oxtoby, D. W. Acc. Chem. Res. 1998, 31, 91-97.

(36) Hutchens, S. B.; Wang, Z.-G. J. Chem. Phys. 2007, 127, art. no. 084912.

(37) Wang, J.; Guo, K.; An, L.; Müller, M.; Wang, Z.-G. Macromolecules 2010, 43, 2037-2041.

(38) Wood, S. M.; Wang, Z.-G. J. Chem. Phys. 2002, 117, 2289-2300.

(39) Wijmans, C. M.; Linse, P. Langmuir 1995, 11, 3748-3756. 